\title{
THE CARDIOVASCULAR GENE ANNOTATION INITIATIVE: IMPACT ON DATA ANALYSIS
}

RC Lovering ${ }^{1 *}$, M Rodriguez-Lopez ${ }^{1}$, NH Campbell ${ }^{1}$, RP Huntley ${ }^{1}$, T Sawford², C O'Donovan ${ }^{3}$, S Orchard ${ }^{4}, \mathrm{H} \mathrm{Hermjakob}^{4}, \mathrm{M} \mathrm{Martin}^{2}, \mathrm{M} \mathrm{Mayr}^{5}$, SE Humphries ${ }^{1}$, PJ Talmud ${ }^{1}$

${ }^{1}$ Institute of Cardiovascular Science, UCL, London, United Kingdom

'UniProt development, European Molecular Biology Laboratory European

Bioinformatics Institute, Hinxton, United Kingdom

${ }^{3}$ UniProt content, European Molecular Biology Laboratory European

Bioinformatics Institute, Hinxton, United Kingdom

${ }^{4}$ Proteomics, European Molecular Biology Laboratory European

Bioinformatics Institute, Hinxton, United Kingdom

${ }^{5}$ Cardiovascular, King's College London, London, United Kingdom

The generation of high-throughput datasets is now a major priority for many research groups. However, often very little thought is given to the essential resources used to analyse these datasets.

The Cardiovascular Gene Annotation Initiative, based at UCL, is one of the groups providing functional annotations to major public biological databases and online functional analysis tools. With our focus on cardiovascular processes we have contributed highly descriptive protein annotations, using the Gene Ontology, which have changed the interpretation of high-throughput datasets, such as transcriptomic, as well as Genome Wide Association studies.

We are now expanding our annotation project to include the annotation of microRNAs and to capture protein-protein interaction data. In the past year we have created over 1000 protein-protein interaction annotations which are accessible via the IntAct and IMEx websites. We will present how this interaction data has substantially increased the annotated networks associated with lipid metabolic and transport processes.

In addition, the interpretation of microRNA data is currently hampered by the lack of consistent, experimentally verified, annotation data: the majority of microRNA annotations are based on predicted data, and the manual annotations available often rely on inconsistent interpretations of experimental data. Consequently, we have begun the process of annotating microRNAs and will present the progress we have made in this area, as well as how this will affect future microRNA research.

Supported by a British Heart Foundation grant RG/13/5/30112.

UCL gene annotation team: www.ucl.ac.uk/functional-gene-annotation.

Gene Ontology: http://geneontology.org.

IntAct: http://www.ebi.ac.uk/intact.

IMEx: http://www.imexconsortium.org. 